\title{
The study of the regularities of structure formation and properties of the L-PBF metal as a set of processes on the way to create a controlled structure
}

\author{
Ivan Shakirov ${ }^{1, *}$, Pavel Kuznetsov ${ }^{1}$, Mikhail Staritsyn ${ }^{1}$, Anton Zhukov ${ }^{1}$ and Vitaliy Bobyr ${ }^{1}$ \\ ${ }^{1}$ NRC "Kurchatov institute"- CRISM "Prometey", Shpalernaya str., StPetersburg 191017, Russia
}

\begin{abstract}
In this work, to study the effect of laser powder bed fusion (LPBF) parameters on the microstructure and mechanical properties of 321 austenitic steel, a series of samples were created combining various combinations of L-PBF technological modes, such as: laser spot diameter, scanning speed, laser power, scanning strategy. The possibility of controlling the structure formation of steel in the L-PBF process with the aim of obtaining a given crystallographic texture, grain size and morphology is estimated. The relationship between the resulting anisotropic structure and mechanical properties is investigated.
\end{abstract}

\section{Introduction}

Layer-by-layer selective laser fusion of the powder layer already finds practical application for the manufacture of products of complex shape. Various studies demonstrate the possibility of growing samples with a geometric shape and internal microstructure, that cannot be obtained using classical technologies based on the mechanical processing of the initial workpiece [1].

This serves as a good signal for designers that they have another kind of "degree of freedom" when designing products of almost any shape. However, the process of fusion of the powder layer occurs when it is sequentially scanned by a laser beam. With this approach, the fused material consists of a large number of microwelded joints in which formed molten bath solidifies under nonequilibrium conditions [2]. Therefore, when changing the geometry of the melt pool, the thermodynamic conditions of crystallization will change, that will lead to the formation of various structures. Based on this, we can say that when changing the scanning parameters, it may be possible to control the structure, that may be another additional "degree of freedom" when creating the product. Thus, it becomes possible to create functional gradient products with a given distribution of properties by volume. For example, you can create an isotropic or anisotropic material, reproduce a predicted structure in a separate volume of a part. This, in turn, creates the prerequisites for the creation of functional products with a heterogeneous structure and untypical properties.

\footnotetext{
* Corresponding author: i.v.shakirov@yandex.ru
} 
Currently, studies of the influence building parameters on the structure of fused steels are already being actively conducted. For example, it was shown in $[3,4]$ that, using various scanning strategies, a clear epitaxial growth along the heat flux with an orientation of $<100>$ can be obtained, that can enhance isotropy. It was also shown that the change in yield strength is mainly associated with different grain sizes, and improved ductility was associated with a changed grain structure, based on the use of different scans [4]. In [5], it was proposed to add to the standard SLM process batch processing using laser shock peening (LSP) in order to increase fatigue properties. Thus, it can be said that despite the accumulated experience in the direction of numerical modeling, the task of assessing the effect of L-PBF regimes on the structure being formed in practice seems relevant. Since, computer modeling simulation results are unable to describe accurately the structure actually obtained on a specific sample at the micro level.

The aim of this work is to study the structure and properties of samples made of stainless austenitic steel powder at various values of the diameter of the focal spot and laser radiation power, scanning speed and the number of repeated scans.

\section{Materials and methods}

Powder of austenitic stainless 321 steel with a dispersion of 0-60 $\mu \mathrm{m}$, made by gas spraying of a melt at Polema JSC, was used as a starting material. The prototypes were made from 321 steel powder by the method L-PBF using the EOSINT M270 setup in which Beam Expander (BE) was installed to change the diameter of the focal spot of laser radiation.

To study the effect of L-PBF parameters on the microstructure and mechanical properties of 321 steel, a series of samples were built combining various combinations of LPBF technological modes, such as laser spot diameter, power, speed, and number of scans. The generalized energy input parameter is calculated as the ratio of the power of the laser source to the speed of movement of the laser spot along the irradiated surface. Three batches of samples were made at various Beam Expander values corresponding to the diameter of the laser spot in the focal plane: 1,2, and 4.5. Within each batch, the samples correspond to the modes presented in the Table 1.

Table 1. L-PBF Parameters.

\begin{tabular}{|c|c|c|c|c|c|}
\hline $\begin{array}{c}\text { Sample } \\
\text { no. }\end{array}$ & $\begin{array}{c}\text { Power, } \\
\mathrm{W}\end{array}$ & $\begin{array}{c}\text { Speed, } \\
\mathrm{mm} / \mathrm{s}\end{array}$ & $\begin{array}{c}\text { Energy input, } \\
\mathrm{W} \cdot \mathrm{s} / \mathrm{mm}\end{array}$ & Scanning \\
\hline 1 & 150.0 & 800 & 100 & 0.188 & \multirow{2}{*}{ Single scan } \\
\hline 2 & 170.0 & 906 & 100 & 0.188 & \\
\hline 3 & 190.0 & 1013 & 100 & 0.188 & \\
\hline 4 & 75.0 & 800 & 50 & 0.094 & \multirow{2}{*}{ Dual scan } \\
\cline { 1 - 5 } 5 & 85.0 & 906 & 50 & 0.094 & \\
\hline 6 & 95.0 & 1013 & 50 & 0.094 & \\
\hline 7 & 112.5 & 800 & 75 & 0.141 & \\
\hline 8 & 127.5 & 906 & 75 & 0.141 & \\
\hline 9 & 142.5 & 1013 & 75 & 0.141 & \\
\hline
\end{tabular}

The modes were chosen in such a way that samples 1-3 were obtained with an energy input of $0.188 \mathrm{~W} \cdot \mathrm{s} / \mathrm{mm}$ taken as $100 \%$, samples $4-6$ with an energy input of $0.141 \mathrm{~W} \cdot \mathrm{s} / \mathrm{mm}$ (50\%), samples 7-9 with an energy input of $0.094 \mathrm{~W} \cdot \mathrm{s} / \mathrm{mm}(75 \%)$, with each sample differing in power and scanning speed. In addition, various scanning modes were used: samples 1-3 single scan, samples 4-9 dual scan. 
The structure was studied using a scanning electron microscope (SEM) VEGA 3 TESCAN. EBSD maps were obtained on a Tescan LYRA 3 microscope with a step of $3 \mu \mathrm{m}$ (grid 400x300 pixels). The density of the samples was determined by hydrostatic weighing on a Mettler Toledo XS105 Excellence XS Analytical scales. Hardness measurements were performed on a Zwick / Roell ZHU 750 top with a load of $10 \mathrm{~N}$. On each sample, 4 measurements were performed.

\section{Results and discussions}

\subsection{Sample density}

The results of density measurement depending on the L-PBF modes are presented in Fig. 1. The figure shows that with the values of Beam Expander 1, 2 and energy inputs of 100 and $75 \%$ for single or dual scanning, respectively, a density of $7.8 \mathrm{~g} / \mathrm{cm}^{3}$ is achieved, that corresponds to a porosity of $1 \%$ or less. With an energy input of $50 \%$, the density of the samples decreases to $7.2 \mathrm{~g} / \mathrm{cm}^{3}$, that corresponds to a porosity of about $10 \%$. For the Beam Expander 4.5 value, it is not possible to achieve a density of more than $7.6 \mathrm{~g} / \mathrm{cm}^{3}$ for any energy input. The lowest density value is $6.7 \mathrm{~g} / \mathrm{cm}^{3}$, that corresponds to a porosity of $15 \%$. The data obtained can be used to create samples with a given value of porosity and more than $15 \%$.

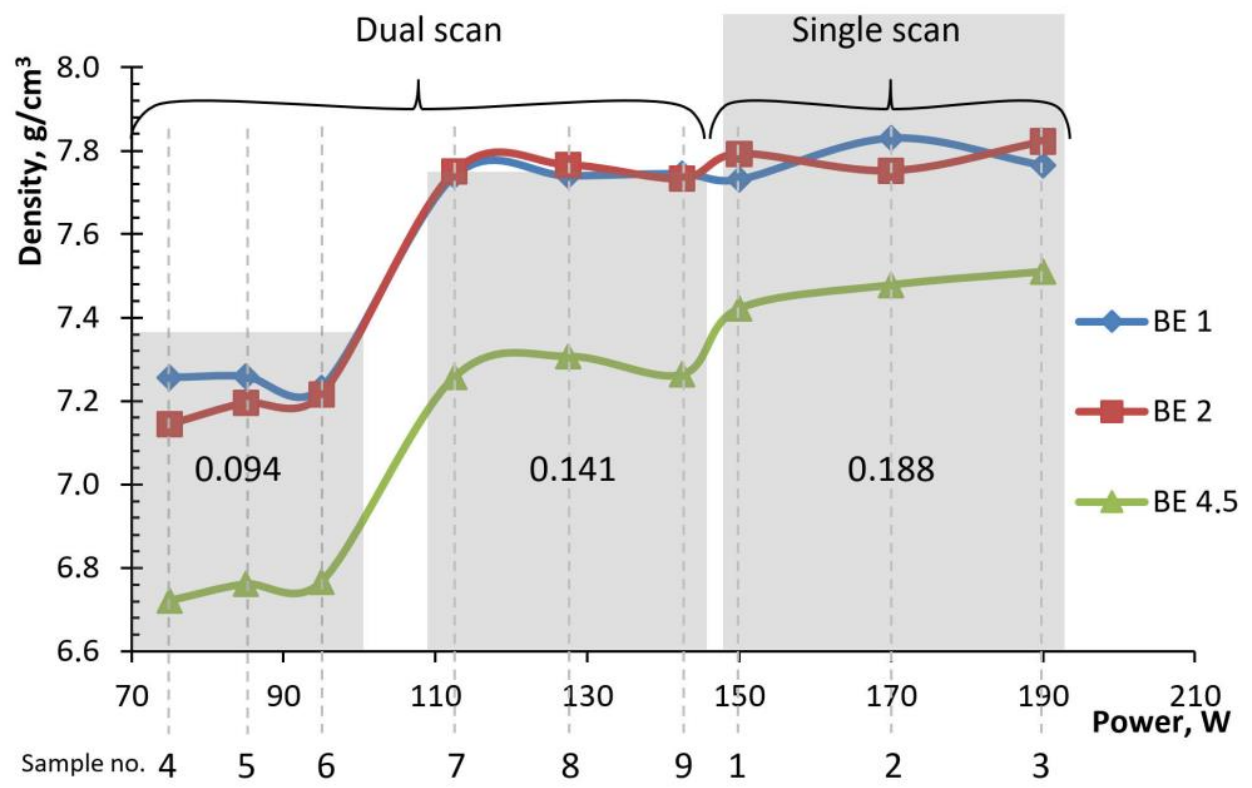

Fig. 1. Dependences of sample density on laser power for various Beam Expander positions.

Thus, we can conclude that the energy input at the level of 100 and $75 \%$ with a single or dual scan and a Beam Expander value of 1 or 2 is sufficient to melt the powder layer and form a high-quality melt bath, which allows to achieve a high density. As the diameter of the laser beam spot increases, an increase in energy input is necessary to achieve the desired density level. 


\subsection{Vickers hardness}

The measured values of the hardness of the samples in a plane parallel to the building direction (BD) are presented in Fig. 2. It can be seen that with a single scan of $100 \%$ energy input, the hardness values are slightly less than with a dual scan at $75 \%$ of the energy input and are 230 and $250 \mathrm{HV}$, respectively. Lower hardness values at the Beam Expander 4.5 position or when scanning at 50\% power are apparently associated with high porosity of the samples.

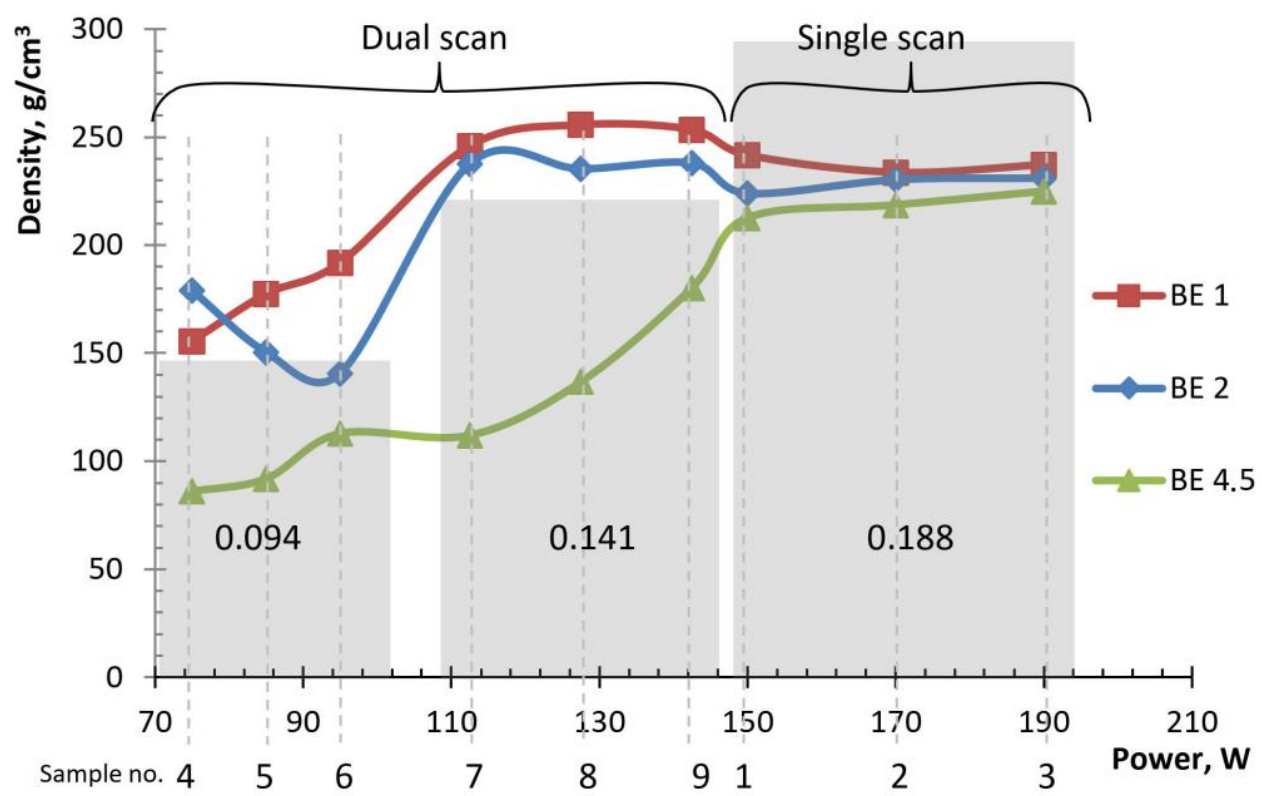

Fig. 2. Dependences of the hardness of the samples on the laser power for different positions of Beam Expander.

The measured hardness values make it possible to evaluate the strength properties of the samples. So, for samples obtained at $100 \%$ energy input, UTS is $800 \mathrm{MPa}$, and for samples obtained at $75 \%$ energy position - $750 \mathrm{MPa}$. This indicates a significant hardening of the samples, which is consistent with the work of other authors [7], in which hardening factors such as dislocation, size, and dispersed particles are noted.

\subsection{Microstructure}

Analysis of the microstructure of the samples by optical metallography, obtained at various Beam Expander values, shows that with an increase in the diameter of the focal spot, the width of the melt pool increases and its depth decreases. For example, Fig. 3 shows the microstructure of the samples obtained in regime №3 at Beam Expander values 1, 2, and 4.5 (see Table 1).

Samples 4-6 show a large number of pores, that corresponds to lower density values (Fig. 1). 
a)
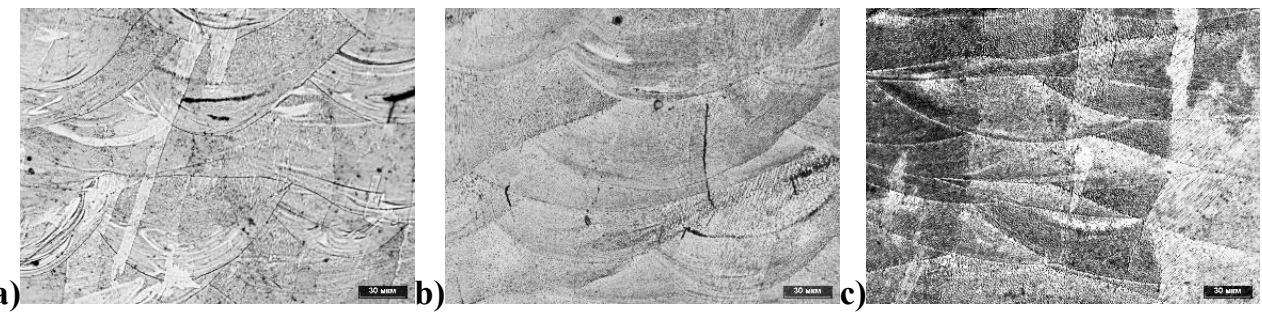

Fig. 3. Optical microscopy. Sample №3, Beam Expander: (a) 1, (b) 2, (c) 4.5.

Micrographs in Fig. 3 show the effect of the focal spot diameter on the solidification structure. The merge lines are clearly visible. The grains consisted of colonies of hardened cells with the same orientation. The grains are drawn parallel to BD and pass through several layers melted by the laser. The average size of the melt bath increases with the increase in the diameter of the laser focal spot with an increase in BE from 1 to 4.5 [6].

a)
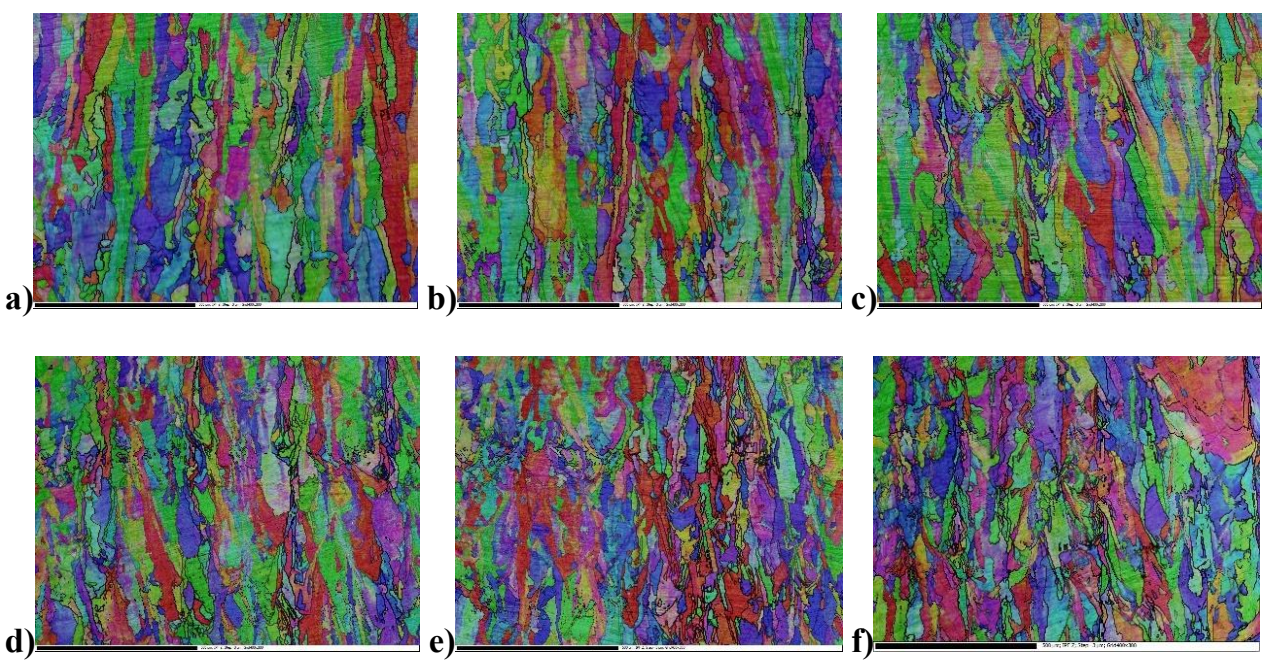

Fig. 4. EBSD maps for sample numbers: (a) 1, (b) 2, (c) 3, (d) 7, (e) 8, (f) 9 and Beam Expander 2.

The structure of samples 1-3 is represented by grains elongated in the sample building direction, spreading through several layers, that indicates their epitaxial growth (see Fig. 4 $a, b, c)$. From the first to the third sample, a proportional increase in power and speed with the constant heat input occurred. In general, this lead to the acceleration of the thermokinetic process of structure formation. Hence, a change occurs from large columnar grains, similar to those formed during casting with directional solidification, to grains with a morphology repeating the shape of the melt baths. The lower rates of metal solidification contribute to the growth of crystallites with the formation of larger grains. With increasing speed, the grain structure is crushed, and crystallization conditions do not favor epitaxial growth. The grains are mainly oriented along the building axis ( $\mathrm{Z}$ axis), and the length of these grains approaches $1 \mathrm{~mm}$, which is much larger than the thickness of the layer used in the construction $(40 \mu \mathrm{m})$.

The use of dual scanning leads to a refinement of the structure. Samples 7-9 with a Beam Expander 2 (see Fig. 4 d, e, f), in comparison with the structure of samples 1-3, have a structure with smaller grains of the order of $100 \mu \mathrm{m}$ in length, whose morphology represented by a drop-shaped form. In the future, dual scanning can be applied in order to refine the structure, to obtain a favorable structure for imparting functional-gradient properties to the whole part or in a separate area of it. 


\section{Conclusion}

In this work, the change in the parameters of the L-PBF process, that is, the diameter of the focal spot, laser radiation power, scanning speed and the number of repeated scans, was evaluated from the point of view of the effect on density, hardness and microstructure. The application of experimental methods contributed to a better fundamental understanding of the correlation between process, microstructure, and properties. The following conclusions can be made:

1) The influence of power and speed was estimated. Increasing the scanning velocity from 800 to $1013 \mathrm{~mm} / \mathrm{s}$ with a proportional increase in power leads to a change in the thermokinetic process of structure formation. A change is observed from large columnar grains, similar to those formed during casting with directional solidification, to grains with morphology of the repeating shape of the melt baths.

2) Dual scanning does not have a summing effect, which affects the physical and mechanical properties of the resulting samples. The use of dual scanning leads to a refinement of the structure. In the future, dual scanning can be applied in order to refine the structure, to obtain a favorable structure for imparting functional-gradient properties to the whole part or in a separate area of it.

3) The diameter of the laser spot has a strong effect on the structure and properties of the resulting sample:

- the low penetration depth of the melt bath, the low ratio between the thermal gradient and the solidification rate, as well as the high cooling rate favor the refinement of the microstructure and a decrease in the texture intensity due to a partial change in the solidification regime from columnar to equiaxial;

- the density directly depends on the energy input - at the level of 100 and $75 \%$ with a single or dual scan and the Beam Expander value of 1 or 2 is enough to melt the powder layer and form a high-quality melt bath. As the diameter of the laser beam spot increases, an increase in energy input is necessary to achieve the desired density level;

The correlations revealed in this work can be effectively used to "tune" the mechanical properties of 321 steel and other austenitic steels on the way to creating functional products with a heterogeneous structure and untypical properties.

This work was supported by the Ministry of science and higher education of the Russian Federation, unique identifier of the agreement RFMEFI62519X0046.

\section{References}

1. P. Köhnen, M. Létang, M. Voshage, J.H. Schleifenbaum, C. Haase, Additive Manufacturing, Understanding the process-microstructure correlations for tailoring the mechanical properties of L-PBF produced austenitic advanced high strength steel, Volume 30, (2019), 100914, ISSN 2214-8604, https://doi.org/10.1016/j.addma.2019.100914.

2. Y. Wang, C. Yu, L. Xing, K. Li, J. Chen, W. Liu, J. Ma, Z. Shen, Journal of Materials Processing Technology, Grain structure and texture of the SLM single track, Volume 281, 116591, (2020), ISSN 0924-0136, https://doi.org/10.1016/j.jmatprotec.2020.116591.

3. Jieren Guan, Yehua Jiang, Xiaowei Zhang, Xiaoyu Chong, Materials Characterization, Microstructural evolution and EBSD analysis of AlSilOMg alloy fabricated by selective laser remelting, Volume 161, 110079, (2020), ISSN 1044-5803, https://doi.org/10.1016/j.matchar.2019.110079. 
4. C.Y. Liu, J.D. Tong, M.G. Jiang, Z.W. Chen, G. Xu, H.B. Liao, P. Wang, X.Y. Wang, M. Xu, C.S. Lao, Materials Science and Engineering: A, Effect of scanning strategy on microstructure and mechanical properties of selective laser melted reduced activation ferritic/martensitic steel, Volume 766, 138364, (2019), ISSN 0921-5093, https://doi.org/10.1016/j.msea.2019.138364.

5. Nikola Kalentics, Manuel Ortega Varela de Seijas, Seth Griffiths, Christian Leinenbach, Roland E. Logé, Additive Manufacturing, 3D laser shock peening - A new method for improving fatigue properties of selective laser melted parts, Volume 33, 101112, (2020), ISSN 2214-8604, https://doi.org/10.1016/j.addma.2020.101112.

6. M. Pham, B. Dovgyy, P.A. Hooper et al. The role of side-branching in microstructure development in laser powder-bed fusion. Nat Commun 11, 749 (2020). https://doi.org/10.1038/s41467-020-14453-3

7. M.V. Staritsyn, P. A. Kuznetsov, S. N. Petrov, M. S. Mikhailov, Physics of Metals and Metallurgy, Composite structure as a strengthening factor for stainless austenitic chromium-nickel additive steel. Volume 121, No. 4, p. 1-7, (2020) (to be published) 